\section{How to manage a high-output stoma}

In this issue Jeremy Nightingale has written an excellent guide on the management of a high-output stoma - authoritative and practical - covering the pathophysiology and practical management. Consider if the stoma is high/output exceeds intake and treat early to avoid complications. Dehydration with water, sodium and magnesium will occur. Remember the sodium content of stoma fluid is usually around $100 \mathrm{mmol} / \mathrm{L}$. The cornerstone of management is to rehydrate and treat thirst avoiding hypotonic fluids. Fluid replacement therefore needs to be with glucose/saline solution (iso-osmolar, the detail is in the paper). Weight and basic biochemistry should be monitored including urinary sodium. Omeprazole to reduce stomal output and loperamide to reduce transit may be helpful. In refractory cases parenteral fluids with added magnesium may be required. Early consideration of restoration of intestinal continuity should be considered where possible. All these issues are discussed in detail including helpful reminders of the basic physiology, dealing with risk factors and complications and a review of longer-term strategies including the potential need for intravenous feeding. Editor's Choice this month. (See page 140)

\section{Provision and standards of care for treatment and follow-up of patients with autoimmune hepatitis (AlH)}

Auto-immune hepatitis is a substantial healthcare burden in the UK with significant variation in care, facilities and opinion regarding management. In this issue Gordon and colleagues report a multi-centre study (28 hospitals) looking at service provision and care of patients. 1/3 lacked a hepatologist, only $18 \%$ had specialist nursing support for Auto-immune Hepatitis and only $40 \%$ employed a histopathologist with a special interest in liver disease. There were significant variations monitoring and treatment $25 \%$ did not continue corticosteroids for 12 months, regular blood monitoring was not always done and not all patients with liver decompensation were referred for consideration of liver transplant. The authors recommend the further development of specialist liver centres as part of regional networks to improve monitoring and care with referral/ discussion of complex cases. There is an excellent accompanying commentary from
Roger Chapman who discusses this and other data that highlight the variable treatment regimens and outcome and need to develop networks of care with therapeutic, organisational and structural changes required to improve the management of patents with autoimmune hepatitis. (See page 126)

\section{Bowel transit studies in children: evidence base, role and practicalities}

Constipation is very common in children, young people and adults. Most have functional constipation and respond well to standard medical therapy. In 'difficult to treat' constipation investigation of colonic function can help to guide therapy. In this issue Popescu and colleagues discuss the strengths and limitations of the commonly available tests including radio-opaque marker studies, scintigraphy, wireless motility capsule and colonic manometry used to assess colonic transit. All address different aspects of colonic function and normal values for many lack an evidence base. The authors comprehensively review the data and provide guidance on the most appropriate test for particular clinical settings - helpfully discussing the specific indications and separating the tests into first and second line. It is great to have all this information pulled together as an invaluable resource when managing difficult constipation and investigation of bowel transit is being considered. (See page 152)

\section{Endoscopic management of intentional foreign body ingestion: experience from a UK centre}

Intentional foreign body ingestion accounts for around $4 \%$ of urgent endoscopic procedures with well recognised complications including impaction and perforation. In this issue Yadollahi and colleagues report their recent experience (1995-2020); 239 episodes in 51 individuals, $78 \%$ female, mean age 23 years, significant increase in incidence during the study period. $223 \mathrm{had}$ a history of psychiatric illness. There was a wide range of different items ingested. $90 \%$ underwent upper gastrointestinal endoscopy, with the item being seen in $88 \%$, mostly in the stomach. Most were removed successfully with mucosal injury seen in 33\% but no other significant complications. In 16 cases the item could not be removed; four managed surgically and eight conservatively. Most procedures were performed between
08.00 and 20.00. There was no association between time of day performed and outcome including length of stay. This is an impressive cohort with service implications, and it is well worth working through the detail in the paper. The authors highlight a number of issues - middle of the night endoscopy can be avoided in most cases, repeat radiology prior to the procedure has the potential to avoid a number of procedures and local protocols should include access to (including investment in) social and psychiatric services. There is an excellent accompanying commentary - Foreign body ingestion: understanding the implications. (See page 98)

\section{Current recommendations on the role of diet in the aetiology and management of IBD}

Diet is a risk factor for and potential treatment option in inflammatory bowel disease. This includes the use of exclusive enteral nutrition as an induction treatment. In this issue Gerasimides and colleagues discuss key themes - the role of diet in disease onset, the indications for and practicalities of exclusive enteral nutrition, partial enteral nutrition as an alternative induction strategy, elimination diets, emulsifiers and additives and the role of supplementary enteral nutrition. The evidence informing some of these issues is at best patchy and the authors call for well designed trials. In an environment where the 'western diet' is clearly a risk factor, inflammatory bowel disease is increasing in incidence and therefore prevalence and there is a high treatment burden optimising diet and nutritional status is crucial, probably best achieved when the condition is stable by healthy eating (which applies to the general population) for overall health and wellbeing. (See page 160)

Please enjoy this issue. Please continue to read, enjoy, and feedback on the journal. Follow us on twitter@FrontGastro_BMJ and listen to our regular podcasts accessed via the journal website https://fg.bmj.com/

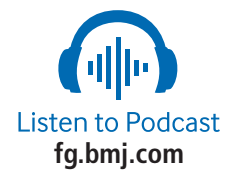

ORCID iD

R Mark Beattie http://orcid.org/00000003-4721-0577 